\title{
Vitamin B6 Effects on Breast Pain in Women of Reproductive Age: A Systematic Review
}

\author{
Roghaieh Rahmani Bilandi (iD) ${ }^{1,}{ }^{*}$ and Asma Salar (iD) ${ }^{1}$ \\ ${ }^{1}$ Department of Midwifery, School of Medicine, Gonabad University of Medical Sciences, Gonabad, Iran \\ "Corresponding author: Department of Midwifery, School of Medicine, Gonabad University of Medical Sciences, Gonabad, Iran. Email: roghaiehrahmany@yahoo.com
}

Received 2020 March 09; Accepted 2020 June 02.

\begin{abstract}
Context: Vitamin B6, which is known as pyridoxine, is used as a nutritional supplement for many therapeutic purposes in medical centers. Women's health is important because they are assumed as the guarantors of health in societies. Breast pain is a common complaint in women of reproductive age and one of the most common reasons for women's referral to health centers. The present study reviewed the impact of vitamin B6 on breast pain.

Evidence Acquisition: In order to gather relevant articles, the databases of IRCT, Iran Doc, SID, MagIran, Medline, Cochrane, Embase, PubMed, Scopus, Science Direct, and Web of Knowledge were searched from 1980 up to July 2019. Keywords were extracted from MeSH, which included "Pyridoxol", "Rodex", "PLP", "Pyridoxal-5-Phosphate", "Pyridoxine Hydrochloride", "Pyridoxine", "Vitamin B6", "Mastodynia", "Mammalgia", "Breast Pain", (Pain AND Breast), "Mastalgia", "Cycle Mastalgia", and "Non-Cycle Mastalgia". The same keywords were also searched in Persian databases. All selected studies had control and intervention groups and compared the painrelieving effects of vitamin $\mathrm{B} 6$ with that of other therapies or placebo.

Results: Among 338 examined articles, seven fulfilled our inclusion criteria and were included in the review. The results showed that vitamin B6 was more effective than placebo in improving breast pain even at low doses.

Conclusions: Vitamin B6 is effective in relieving breast pain. However, there are also other supplements and medications to treat breast pain, so some factors like cost-effectiveness, availability, and side effects should be considered to choose a suitable agent.
\end{abstract}

Keywords: Vitamin B6, Pyridoxine, Mastalgia, Breast Pain

\section{Context}

Women's health is one of the priorities of any society because they are more vulnerable than men, and their health guarantees the family's and society's well-being (1, 2). Most women experience breast pain as a common phenomenon throughout their lives; nevertheless, it is usually benign. Women's fear of malignant breast tumors is one of the main reasons for their referral to therapeutic and counseling centers (3). However, it should be noted that mastalgia is an uncommon symptom of cancer (4). Breast pain accounts for more than $50 \%$ of outpatient referrals to medical centers (5). The prevalence of mastalgia, which is defined as moderate to severe breast pain for more than five days, is $69 \%$. Breast pain can interfere with somatic, physical, and social activities (6) and is the most common physical symptom of premenstrual syndrome (7). Many painrelieving agents such as nonsteroidal anti-inflammatory drugs (NSAIDs), Danazol or Mefenamic acid, and supplements like vitamin $\mathrm{B} 6$, vitamin $\mathrm{E}$, and herbal remedies have been tried to manage breast pain $(7,8)$.
Vitamin B6, or pyridoxine, plays an important role in the body's catabolism and anabolism reactions (9). The body's active pyridoxine form is pyridoxal-5-phosphate (PLP) coenzyme that has a key role in the metabolism of amino acids and nucleic acids, as well as in biological pathways (e.g., gluconeogenesis) (10) and protection of deoxyribonucleic acid against damage (11). Some studies suggest vitamin $\mathrm{B} 6$ be useful in treating premenstrual symptoms, believing that this vitamin increases serotonin synthesis, a neurotransmitter that is effective in treating depression (7). In addition, it has recently been suggested that vitamin B6 is essential for the synthesis of prostaglandins and fatty acids, which is an additional role to its previously reported effects in correcting the estrogen metabolism disorder in the context of premenstrual syndrome $(12,13)$. In one study, the positive effects of vitamin B6 in reducing the symptoms of depression, anxiety, and breast pain have been confirmed (14). In contrast, some studies have shown no painrelieving effects for this vitamin in the case of breast pain (15). 
Nowadays, studies have highlighted the role of vitamin B6 in improving breast pain through its effect on molecular, cellular, and biochemical processes (such as regulating the synthesis of brain monoamines, fatty acids, and prostaglandins). Moreover, due to its negligible complications and widespread availability, it is interesting to ask if there is sufficient evidence to support the beneficial effects of vitamin B6 for managing breast pain. In addition, the negative impacts of mastalgia on women's health have been identified. The lack of a comprehensive systematic study on the role of pyridoxine in treating mastalgia led us to conduct this review in order to investigate the impact of vitamin B6 supplementation on breast pain.

\section{Evidence Acquisition}

The present study was a systematic review to divulge the effectiveness of vitamin B6 in managing breast pain. The PRISMA checklist (2009) was used to conduct the review (16). The review was done in four stages, including designing the study and determining the search strategy, collecting articles, evaluating inclusion and exclusion criteria, and finally, the qualitative evaluation of articles.

Keywords were extracted from the MeSH, and searches were performed in the databases of IRCT, Iran Doc, SID, and MagIran for Persian articles and Medline, Cochrane, Google Scholar, Embase, PubMed, Scopus, Science Direct, and Web of Sciences for English articles from 1980 up to July 2019. The keywords included "Pyridoxol", "Rodex", "PLP", "Pyridoxal-5-Phosphate", "Pyridoxine Hydrochloride", "Pyridoxol Hydrochloride", "Vitamin E", "Pyridoxine", "Vitamin B6", "Vitamin B 6", "Mastodynia", "Mammalgia", "Breast Pain", "Breast Pains", (Pain AND Breast), "PMS", "Premenstrual Syndrome mastalgia", "Mastalgia", "Cycle Mastalgia", and "Non-Cycle Mastalgia".

The search was conducted by two experts, and 338 articles were found after the primary search. Upon an initial check, 54 repetitive articles were removed. After reading titles and abstracts, 243 irrelevant articles and 8 articles were the sampling stage which recorded at Cochrane Library database and four articles that had no full texts, were removed. Finally, 29 related articles were analyzed. The study selection process was performed according to the PRISMA flowchart (17).

Inclusion criteria were conducting the study on the women of reproductive age with cyclic mastalgia, having a clinical or semi-experimental design, and investigating the effects of vitamin B6 on mastalgia. The studies conducted on breast tumors, case reports, case series, the studies assessing vitamin B6 effects with other therapies, and conference statements were not included. Also, the studies that hadn't full texts, those with a sample size of fewer than 20 subjects per group, and the PMS (premenstural syndrom) studies that had not reported breast pain were removed.

From 29 articles, four studies due to a sample size of less than 20 in each group, four articles due to the simultaneous use of vitamin B6 and another supplement, and two articles due to the lack of transparency in results and statistical analyses were removed from the study. In addition, 11 articles performed on women with premenstrual syndrome were removed as they had not mentioned breast pain as a premenstrual symptom in their findings. Finally, seven articles entered the final review process. The data of these articles, including the first author's name, year of publication, control group, age of participants, study design, sample size after randomization, patients' characteristics, treatment period, blinding process, details of the intervention, measurement methods, statistical tests, and their findings were extracted (Figure 1).

In order to assess the quality of the articles, the Jadad Scale was used $(18,19)$, which included five questions about randomization, the probability of bias, Study blindness, following up of patients, and the criteria of the discontinuation of the trial. Answering "Yes" to each question was scored one while "No" was scored zero. The maximum score is 5 , and the minimum is zero. The processes of selecting and qualifying articles were done by two of the authors. Disagreements were resolved through group discussions. The articles' qualitative evaluation scores have been shown in Table 1.

\section{Results}

Four out of seven articles exclusively addressed breast pain, including one study without a control group, two studies on vitamin B6 supplementation with evening primrose and vitamin E, respectively, and one placebocontrolled trial. The other three studies described the synergistic effects of vitamin B6 with either placebo, a chemical drug, or a non-pharmacological method in women with mastalgia premenstrual syndrome (Table 2).

Soltani et al. (2016) compared the effects of vitamin B6 and placebo on breast pain in 72 women; they administered vitamin B6 (100 mg) twice a day for two months. The prevalence and severity of pain was more reduced in the vitamin B6 group compared with the placebo group. Mean baseline pain scores were 1.51 in control and 1.67 in the intervention group, showing 0.75 and 0.61 reduction after treatments, respectively $(\mathrm{P}<0.01)(20)$.

Jahdi et al. (2018) compared the effects of vitamin B6 and evening primrose. Thirty-one women ( 18 - 50 years old) received $50 \mathrm{mg}$ vitamin $\mathrm{B} 6$ twice daily for three months. The results showed that both evening primrose oil (EPO) 


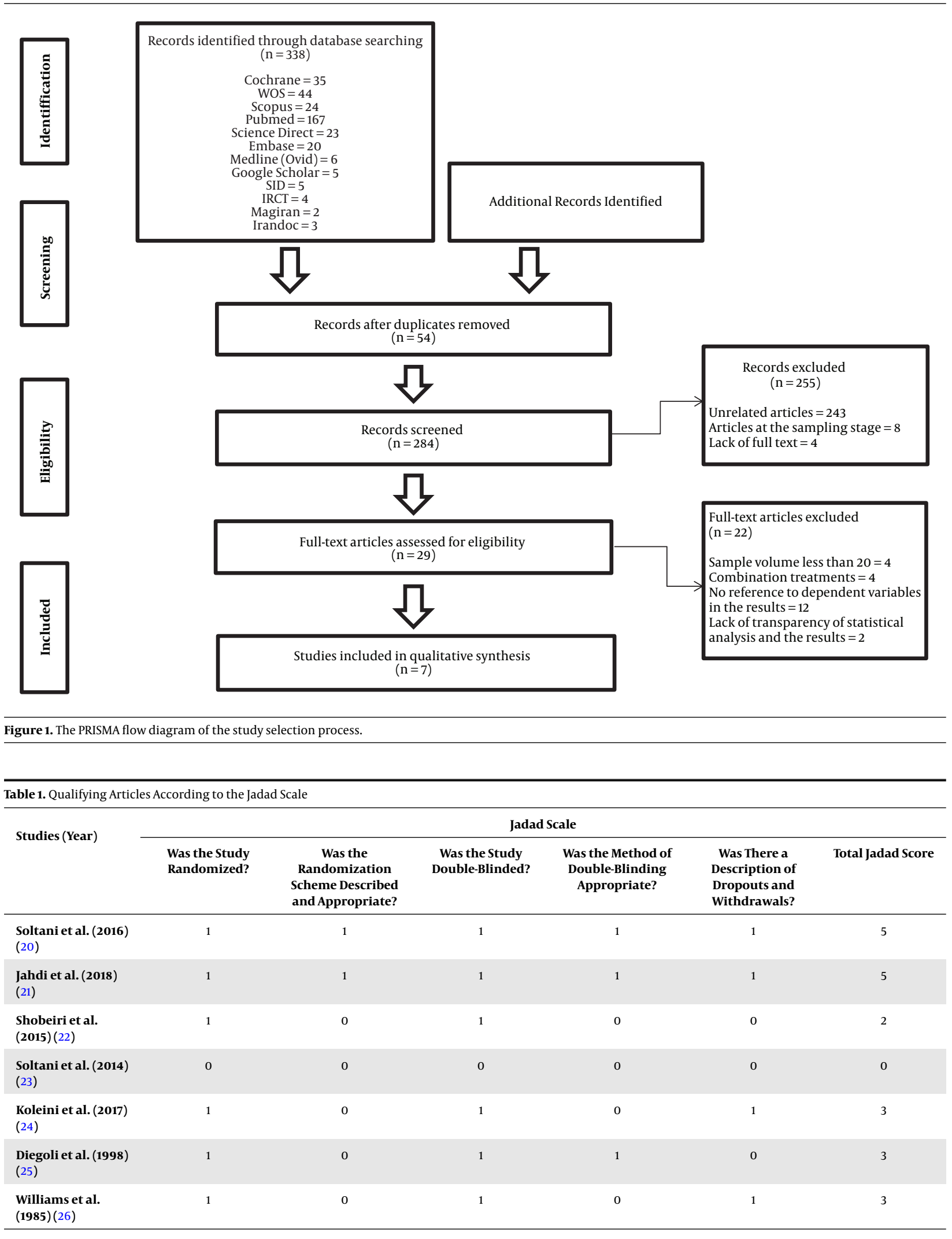




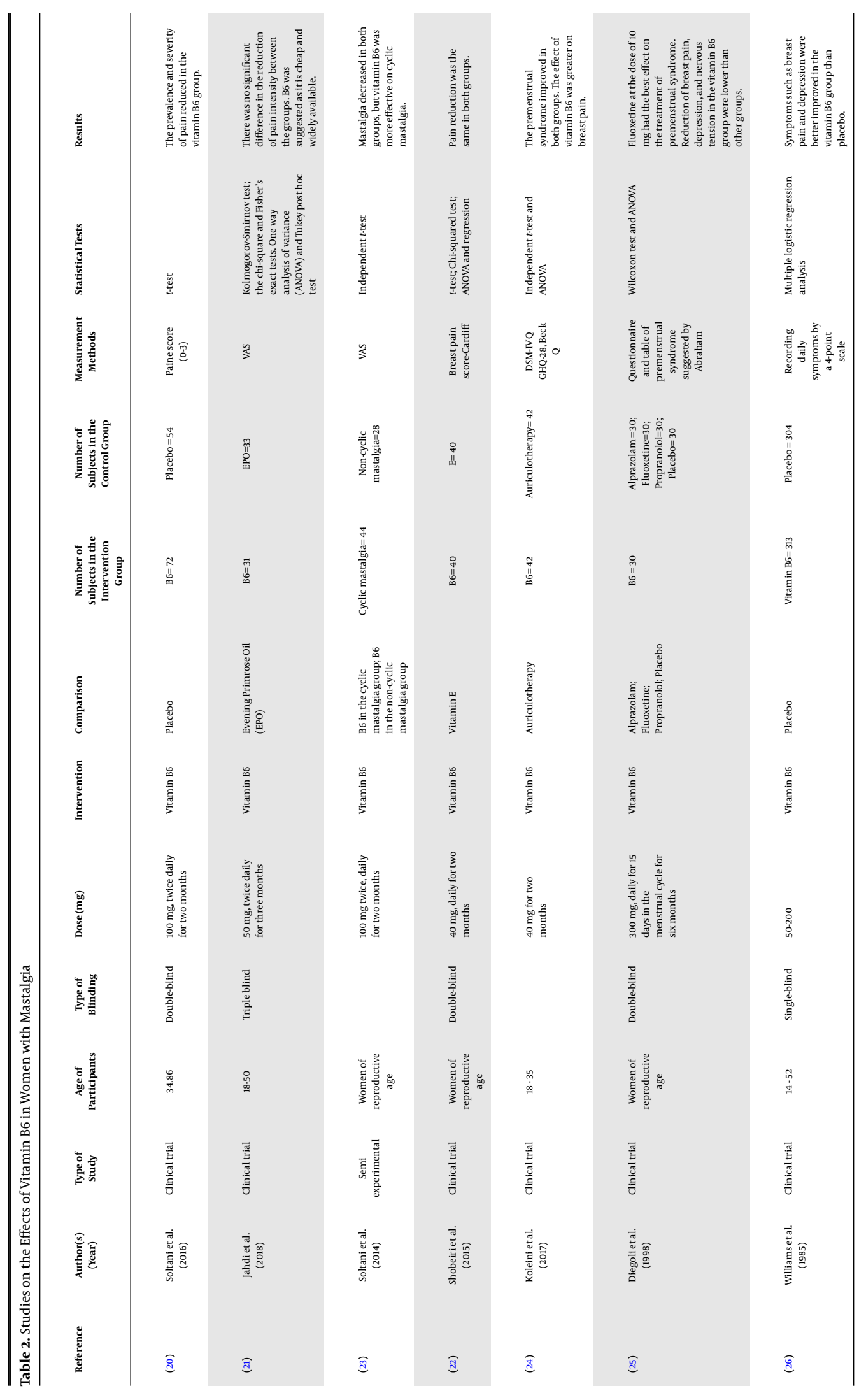


and vitamin B6 decreased breast pain, but vitamin B6, as a cheaper and more easily available agent, was shown to have significantly better pain-reducing effects $(\mathrm{P}<0.001$ and $\mathrm{CI}=95 \%$ ). In the placebo group, there was no significant reduction in breast pain $(\mathrm{P}=0.815)(21)$.

Soltani et al. (2014), in their study, assessed women of reproductive age with cyclic and non-cyclic breast pain. They prescribed $100 \mathrm{mg}$ vitamin B6 twice every day for two months. The participant's characteristics (age, mean pain score, and the prevalence of pain) were similar in both groups $(\mathrm{P}>0.05)$. Pain reduction was observed in both groups; however, vitamin B6 was more effective in treating women with cyclic breast pain $(\mathrm{P}<0.01)(23)$.

Shobeiri et al. (2015) compared the effects of vitamin B6 (40 mg for two months) and vitamin E in a study on 40 women of childbearing age with breast pain. The same pain reducing effectiveness was observed in the both groups $(\mathrm{P}<0.001)$. Both interventions also showed similar efficiency for treating breast pain $(\beta=0.807$, standard error $(S E)=0.433, P=0.067)(22)$.

Koleini et al. (2017) compared the effects of vitamin B6 with those of auriculotherapy. Forty mg vitamin B6 was given to 42 women (18 - 35 years old) with premenstrual syndrome for two months, revealing the positive effects of this vitamin on breast pain. Mastalgia frequency significantly decreased in the vitamin B6 compared with auriculotherapy group (46.2\% vs. $23.1 \%, \mathrm{P}=0.001 \& \mathrm{CI}=95 \%$ ) (24).

Diegoli et al. (1998) gave $300 \mathrm{mg}$ vitamin B6 to women with premenstrual syndrome during their menstrual cycles, and then compared breast pain with those received alprazolam, fluoxetine, and propranolol, given every day for 15 days. Each group received three months of placebo and three months of intervention. The best outcome was observed at the $10 \mathrm{mg}$ dose of fluoxetine while vitamin B6 had poor effectiveness compared with other groups (breast pain reduction of $49 \%$ (vitamin B6) versus $67.2 \%$ (fluoxetine), $\mathrm{P}=0.0001)$. Covariation analysis found a significant difference between the pyridoxine group and the others $(\mathrm{P}=0.025)(25)$.

Williams et al. (1985) prescribed 50 - $200 \mathrm{mg}$ vitamin B6 to 313 women with premenstrual syndrome and compared its pain reducing effects with a placebo. Logistic regression showed that vitamin B6 was more effective than the placebo in improving premenstrual mastalgia syndrome $(\mathrm{P}<0.02)$. Also, the effectiveness of vitamin B6 on mastalgia increased at higher doses of the vitamin (26).

\section{Discussion}

This study evaluated the effects of vitamin B6 on breast pain. So far, no comprehensive systematic review has ad- dressed this issue. For this purpose, we evaluated controlled blinded clinical trials.

The pain reducing effects of vitamin B6 can be related to increased production of serotonin and dopamine, as well as the synthesis of prostaglandins and essential fatty acids. Collectively, these biological effects of vitamin B6 may contribute to its role in improving mastalgia. Multiple studies have confirmed the efficiency of vitamin B6 in reducing breast pain $(20,22,24)$. In addition, it has been noted that vitamin $\mathrm{B} 6$ reduces serum renin and aldosterone, sodium and water reabsorption, and edema, improving physical symptoms including breast pain (12).

The effect of vitamin B6 on reducing breast pain has been compared with vitamin $\mathrm{E}$ and a herbal medicine (i.e., EPO), revealing the same efficacy for all three agents $(21,22)$. In the study of Jahdi et al., vitamin B6 was tested against EPO, vitamin B6 was recommended because of its availability and cheapness (21). Other studies have also compared the analgesic effects of vitamin E with that of EPO (27-33), and some have reported comparable effects for the two agents in reducing breast pain $(27,29,31,33)$. In one study, however, the effects of EPO were more significant than those of vitamin E (28). The recent study was conducted on women with premenstrual syndrome, while the aforementioned studies specifically examined breast pain changes, which may explain the inconsistency between their findings.

Vitamin B6 has been noted to be more effective than non-pharmacological methods (such as auriculotherapy) in alleviating breast pain (24). Auriculotherapy is a common Chinese medicine approach in which specific points on the auricle are stimulated to treat various disorders. Different tools such as acupuncture and press tack needles can be applied on the auricular points located on the external ear (34). In one study, the effects of auriculotherapy were superior versus placebo on back pain (35). In the study of Koleini et al, pyridoxine showed greater effects on breast pain compared with auriculotherapy (24).

In a study by Soltani et al., vitamin B6 was more effective than a placebo in reducing breast pain (20). The efficacy of minimal doses of vitamin B6 versus placebo was also confirmed in another study (26). In contrast, comparing the pain-reducing effects of vitamin B6, A, and D and placebo showed no significant differences between the groups in patients with breast pain (15). All three studies were blinded trials and had large sample sizes; however, this inconsistency may be due to different measurement tools applied by them (a qualitative tool was used in the recent study). In addition, researchers in the recent report had used a combination of vitamins.

In the only study on women with breast pain during the menstrual cycle, $300 \mathrm{mg}$ vitamin B6 was daily pre- 
scribed versus fluoxetine for 15 days during a three-month period. Fluoxetine had better effectiveness in improving breast pain compared with vitamin B6 (25). Fluoxetine is a common drug used for treating premenstrual syndrome and one of the most common therapeutics for improving PMS mood symptoms (36). However, it is associated with complications such as insomnia, restlessness, and reduction of sexual desire (37). While various studies have noted lower complications for vitamin B6 compared to other drugs, one study reported some complications for vitamin B6 (20\%), which was lower than the complications of bromocriptine (65\%) (38).

\subsection{Conclusions}

The effects of vitamin B6 on breast pain were superior versus placebo and non-pharmacological methods. Vitamin $\mathrm{B} 6$ also showed its beneficial effects even at low doses (e.g., $40 \mathrm{mg}$ ). Although the positive effects of other drugs have been noted on improving mastagia, their complications, availability, and cost-effectiveness should also be taken into account in order to select a suitable drug.

\section{Footnotes}

Authors' Contribution: Study concept and design: R. R. and A. S.; Acquisition of data: A. S.; Analysis and interpretation of data: R. R. and A. S.; Drafting the manuscript: A. S.; Critical revision of the manuscript for important intellectual content: R. R.; Administrative, technical, and material support: A. S., Study supervision: R. R.

Conflict of Interests: There is no conflict of interest.

Funding/Support: The authors appreciate all the researchers whose articles were used in this systematic review.

\section{References}

1. Seyedfatemi N, Salsali M, Rezaee N, Rahnavard Z. Women's health concept: A meta-synthesis study. Iran J Public Health. 2014;43(10).

2. Sepehrmanesh Z. [Mental health and its related factors in young women in Kashan City]. Iran J Obstet Gynecol Infertil. 2009;12(1):31-41. Persian.

3. Shobeiri F, Oshvandi K, Nazari M. Cyclical mastalgia: Prevalence and associated determinants in Hamadan City, Iran. Asian Pac J Trop Biomed. 2016;6(3):275-8. doi:10.1016/j.apjtb.2015.12.008.

4. Eren T, Aslan A, Ozemir IA, Baysal H, Sagiroglu J, Ekinci O, et al. Factors effecting mastalgia. Breast Care (Basel). 2016;11(3):18893. doi: 10.1159/000444359. [PubMed: 27493619]. [PubMed Central: PMC4960349].

5. Hafiz SP, Barnes NLP, Kirwan CC. Clinical management of idiopathic mastalgia: A systematic review. J Prim Health Care. 2018;10(4):312-23. doi:10.1071/HC18026. [PubMed: 31039960].

6. Sheidaei S, Irani M, Ghazanfarpour M. [The effect of herbal medicines and supplements on mastalgia: A systematic review and metaanalysis of clinical trials in Iran]. Iran J Obstet Gynecol Infertil. 2019;22(3):87-98. Persian. doi: 10.22038/ijogi.2019.13397.
7. Shayan AAH, Masumee Z, Shobeiri F, Moradkhany SH, Sureenejad H. [Evaluation of the effect of vitamin B6 and primrose oil on the symptoms of premenstural syndrom: Randomized clinical trial]. Iran J Obstet Gynecol Infertil. 2019;21(12). Persian.

8. Hajizadeh K, Alizadeh Charandabi SM, Hasanzade R, Mirghafourvand M. Effect of vitamin E on severity and duration of cyclic mastalgia: A systematic review and meta-analysis. Complement Ther Med. 2019;44:18. doi: 10.1016/j.ctim.2019.03.014. [PubMed: 31126540].

9. Zhao LG, Shu XO, Li HL, Gao J, Han LH, Wang J, et al. Prospective cohort studies of dietary vitamin B6 intake and risk of cause-specific mortality. Clin Nutr. 2019;38(3):1180-7. doi: 10.1016/j.clnu.2018.04.016. [PubMed: 29764693]. [PubMed Central: PMC6551204].

10. Zhao M, Lamers Y, Ralat MA, Coats BS, Chi YY, Muller KE, et al. Marginal vitamin B-6 deficiency decreases plasma (n-3) and (n-6) PUFA concentrations in healthy men and women. J Nutr. 2012;142(10):17917. doi: 10.3945/jn.112.163246. [PubMed: 22955512]. [PubMed Central: PMC3442793].

11. Kuwahara K, Nanri A, Pham NM, Kurotani K, Kume A, Sato M, et al. Serum vitamin B6, folate, and homocysteine concentrations and oxidative DNA damage in Japanese men and women. $\mathrm{Nu}$ trition. 2013;29(10):1219-23. doi: 10.1016/j.nut.2013.03.014. [PubMed: 23800563].

12. Valiani M, samadi Z, shadman F. [Comparison the effects of aerobic exercise and vitamin B6 in severity of symptoms of premenstrual syndrome in non-athlete girls]. Complementary Medicine Journal. 2013;3(3):552-62. Persian.

13. Kiani F, Sayehmiri K, Sayehmiri F, Naghdi N, Ghafari M, Asadi-Samani $M$, et al. Effects of vitamin B6 on premenstrual syndrome: A systematic review and meta-analysis. J Chem Pharm Res. 2016;9(3):1346-53.

14. Salehi L, Salehi F. The effect of pyridoxine (vit B6) on premenstrual syndrome. J kordestan Univ Med Sci. 2007;2(3):32-9.

15. De Luca LA, Goncalves Mde F, de Carvalho LR. [Pre-menstrual cyclic mastalgia]. Rev Assoc Med Bras (1992). 2006;52(4):265-9. doi: 10.1590/s0104-42302006000400029. [PubMed: 16967147].

16. Moher D, Liberati A, Tetzlaff J, Altman DG; The PRISMA Group. Checklist prisma. 2009, [cited 17 Feb 2020]. Available from: http://www.prismastatement.org/.

17. Moher D, Liberati A, Tetzlaff J, Altman DG; The PRISMA Group. PRISMA Flow Diagram. 2009, [cited 1 Dec 2019]. Available from: http://www. prisma-statement.org/.

18. Bhandari M, Richards RR, Sprague S, Schemitsch EH. Quality in the reporting of randomized trials in surgery: is the Jadad scale reliable? Controlled clinical trials. Control Clin Trials. 2001;22(6):687-8. doi: 10.1016/s0197-2456(01)00147-7.

19. PMIDCAL. Oxford quality scoring system, Jadad scale. 2020, [cited $16 \mathrm{Feb}$ 2020]. Available from: http://www.pmidcalc.org/.

20. Soltany S, Alavy Toussy J, Gholamaliyan E. [Effect of vitamin B6 on mastalgia: A double blind clinical trial]. Koomesh. 2016;17(4):950-6. Persian.

21. Jahdi F, Tolouei R, Neisani Samani L, Hashemian M, Haghani H, Mojab F, et al. Effect of evening primrose oil and vitamin $\mathrm{B} 6$ on pain control of cyclic mastalgia associated with fibrocystic breast changes: A triple-blind randomized controlled trial. Shiraz E Med J. 2018;20(5). e81243. doi: $10.5812 /$ semj.81243.

22. Shobeiri F, Oshvandi K, Nazari M. Clinical effectiveness of vitamin E and vitamin B6 for improving pain severity in cyclic mastalgia. Iran J Nurs Midwifery Res. 2015;20(6):723-7. doi: 10.4103/1735-9066.170003. [PubMed: 26793260]. [PubMed Central: PMC4700694].

23. Soltany S, Alavy Toussy J. The effect of vitamin B6 on cyclic and noncyclic mastalgia. Adv Environ Biol. 2014:2936-9.

24. Koleini S, Valiani M. Comparing the effect of auriculotherapy and vitamin B6 on the symptoms of premenstrual syndrome among the students who lived in the Dorm of Isfahan University of Medical Sciences. Iran J Nurs Midwifery Res. 2017;22(5):354-8. doi: 10.4103/ijnmr.IJNMR_120_16. [PubMed: 29033988]. [PubMed Central: PMC5637142]. 
25. Diegoli MSC, da Fonseca AM, Diegoli CA, Pinotti JA. A double-blind trial of four medications to treat severe premenstrual syndrome. Int J Gy necol Obstet. 1998;62(1):63-7. doi: 10.1016/s0020-7292(98)00035-6.

26. Williams MJ, Harris RI, Dean BC. Controlled trial of pyridoxine in the premenstrual syndrome. J Int Med Res. 1985;13(3):174-9. doi: 10.1177/030006058501300305. [PubMed:3891456].

27. Alvandipour M, Tayebi P, ALIZADEH NR, Khodabakhshi H. [Comparison between effect of evening primrose oil and vitamin $\mathrm{E}$ in treatment of cyclic mastalgia]. J Babol Univ Medical Sci. 2011;13(2 (59)):7-11. Persian.

28. MASOUMI SZ, Khalili A, Delforooz A, Faradmal J, Shayan A. [Comparison the effect of evening primrose oil and vitamin $\mathrm{E}$ on premenstrual syndrome]. Complementary Medicine Journal. 2017;7(2 (23)):193143. Persian.

29. Salehi A, Momeni H, Seraji A. [The effect of evening primrose and vitex on cyclic mastalgia in comparison with vitamin E: a randomized clinical trial]. Yafteh. 2013;15(2):95-109. Persian.

30. Jaafarnejad F, Adibmoghaddam E, Emami SA, Saki A. Compare the effect of flaxseed, evening primrose oil and Vitamin $\mathrm{E}$ on duration of periodic breast pain. J Educ Health Promot. 2017;6:85 doi: 10.4103/jehp.jehp_83_16. [PubMed: 29114553]. [PubMed Central: PMC5651668].

31. Fathizadeh N, Takfallah L, Ehsanpour S, Namnabati M, Askari S. [Effects of evening primrose oil and vitamin $\mathrm{E}$ on the severity of periodical breast pain]. Iran J Nurs Midwifery Res. 2009;13(3). Persian.

32. Jafarnezhad F, Adib Moghaddam E, Ahmad Emami S, Saki A, Ham
M, Mohammadzadeh Vatanchi A. [Comparative effect of flaxseed and evening primrose oil with vitamin $\mathrm{E}$ on severity of cyclic mastalgia in women]. Iran J Obstet Gynecol Infertil. 2016;19(22):8-16. Persian.

33. Pruthi S, Wahner-Roedler DL, Torkelson CJ, Cha SS, Thicke LS, Hazelton $\mathrm{JH}$, et al. Vitamin E and evening primrose oil for management of cyclical mastalgia: a randomized pilot study. Altern Med Rev. 2010;15(1):59.

34. Suen LKP, Molassiotis A, Yueng SKW, Yeh CH. Comparison of magnetic auriculotherapy, laser auriculotherapy and their combination for treatment of insomnia in the elderly: A double-blinded randomised trial.Evid Based Complement Alternat Med.2019;2019:3651268. doi: 10.1155/2019/3651268. [PubMed: 31239857]. [PubMed Central: PMC6556291]

35. Mafetoni RR, Shimo AK. Effects of auriculotherapy on labour pain: a randomized clinical trial. Rev Esc Enferm USP. 2016;50(5):726-32. doi: 10.1590/S0080-623420160000600003. [PubMed: 27982389].

36. Mohajerani M, Rezvani A. [Comparison of the effect of herbal medicine(Flint) with fluoxetine in the management of premenstrual syndrome in patients referred to Islamic Azad University clinics of Mashhad]. Scientific Journal Management System. 2015;6(2):1-8. Persian.

37. Sabet-Birjandi S, Tadayon-Najafabadi M, Siyahpooshan A, Haghighizadeh M. [Comparision the effect of hypericum perforatum and vitamin B6 in the treatment of premenstrual syndrome]. Zahedan J Res Med Sci. 2011;13(4):1-5. Persian.

38. Sharma P, Kulshreshtha S, Singh GM, Bhagoliwal A. Role of bromocriptine and pyridoxine in premenstrual tension syndrome. Indian J Physiol Pharmacol. 2007;51(4):368-74. [PubMed:18476391]. 